\title{
Zonificación del potencial energético de la biomasa residual forestal en la cuenca del lago Ranco, Chile. Antecedentes para la planificación energética regional
}

\author{
Zoning the energy potential of forest residual biomass in the watershed of Ranco Lake. Background \\ for regional energy planning
}

\author{
Oscar Torres-Álvarez a*, Fernando Peña-Cortés ${ }^{\text {a }}$ \\ *Autor de correspondencia: ${ }^{a}$ Universidad Católica de Temuco, Facultad de Recursos Naturales, Escuela de Ciencias Ambientales, \\ Laboratorio de Planificación Territorial, Temuco, Chile, tel. 45-205469, casilla 15-D, otorres@uct.cl
}

\begin{abstract}
SUMMARY
Land planning comprises a stage of land management whose aim is to generate proposals from analyzing the information of the territory, which represents multiple variables of the geographic space. Some of the most recent lines of planning are renewable energies and particularly residual forest biomass since this is a non used environmental element but with a high growth potential. For this task, using technological tools like geographic information systems or remote sensing permits to handle these topics and express them in a spatial zoning for decision making. According to this, a prospective work was made in the watershed of Ranco Lake, where optimum spaces were defined to obtain residual forest biomass in four vegetational units (coigüe-raulí-tepa, roble-raulí-coigüe, evergreen and forest plantations). The results showed that there exists an overall potential surface to handle of 45,555 hectares, from which the forest type coigüe-raulí-tepa highlights with a $62.4 \%$ of the surface. Under the assumption of availability of residual forest biomass in the watershed and a scene of energetic crisis, the importance of prioritizing regional policies and research concerning the development of non conventional renewable energies is recognized.
\end{abstract}

Key words: territorial zoning, GIS and remote sensing, residual forest biomass, NCRE.

\section{RESUMEN}

La planificación territorial constituye una etapa del ordenamiento del territorio que tiene por objeto generar propuestas a partir del análisis de información obtenida de múltiples variables del espacio geográfico. Algunas de las líneas más recientes de planificación la constituyen las energías renovables, y entre éstas la biomasa residual forestal, puesto que constituye un pasivo ambiental no utilizado pero con gran potencial de crecimiento. En esta tarea, el uso de herramientas tecnológicas como el sistema de información geográfica y la percepción remota permiten manejar información territorial asociada a la biomasa residual forestal y expresarla en una zonificación espacial para la toma de decisiones. En este marco, se efectuó un trabajo prospectivo en la cuenca del lago Ranco donde se determinaron los espacios potenciales de extracción de biomasa residual forestal en cuatro unidades de vegetación (coigüe-raulítepa, roble-raulí-coigüe, siempreverde y plantaciones forestales) bajo las actuales condiciones de cosecha. Los resultados mostraron que existe una superficie potencial total a manejar de 45.555 ha, de éstas el tipo forestal coigüe-raulí-tepa, con 62,4\% de la superficie, representó la mayor proporción. Bajo el supuesto de la actual disponibilidad de biomasa residual forestal en la cuenca y un escenario de crisis energético, se reconoce la importancia de priorizar las políticas regionales y la investigación en torno al desarrollo del sector de las energías renovables no convencionales.

Palabras clave: zonificación territorial, SIG y percepción remota, biomasa residual forestal, energías renovables no convencionales.

\section{INTRODUCCIÓN}

La planificación territorial constituye una etapa del ordenamiento territorial que tiene por objeto generar propuestas a partir del análisis de información del territorio para regular y gestionar las actividades implicadas en las transformaciones territoriales, siendo su objetivo entregar una relación coordinada entre el hombre y el medio ambiente (Gómez-Orea 2007). En esta actividad los nuevos instrumentos de análisis geográfico pueden cumplir diferentes funciones en las distintas etapas de la planificación territorial. Precisamente, el uso de herramientas para pla- nificar el espacio geográfico, genera un abanico de actividades, desde la descripción y gestión, hasta la ordenación y planificación del territorio.

Un punto relevante en este proceso es contar con información territorial expresada como un conjunto de datos actualizados sobre una región específica que facilite la toma de decisiones (Peña-Cortés et al. 2009). Principalmente, por que esta última se ve influenciada por el tipo y cantidad de información territorial ya disponible, el tamaño de la zona, la legislación y las posibilidades de recopilar nuevos datos para adaptarse a las necesidades (Geneletti y Van Duren 2008). Así, la información territorial es conse- 
cuencia y motor de desarrollo, y es la base para la materialización de proyectos, programas y nuevas oportunidades de inversión en un espacio geográfico determinado (Chen et al. 2008).

En esta línea, para el procesamiento de la información territorial existen herramientas tecnológicas, como los sistemas de información geográfica (SIG) y la percepción remota, que pueden almacenar, analizar y vincular espacialmente las distintas fuentes de información territorial provenientes de múltiples sectores (Belmonte et al. 2008, Peña-Cortés et al. 2009). En consecuencia, los sistemas de información geográfica pueden entenderse como una caja de experimentación que permite al gestor territorial trabajar o plantearse diferentes escenarios de una determinada región: por una parte, los que se producirían con la ejecución de ciertas políticas, o los que ocurrirían siguiendo determinadas tendencias. Una aplicación que muestra la importancia de estas herramientas es la zonificación territorial que, visto desde un marco ecológico y productivo, permite la identificación y delimitación de las principales zonas de importancia para la conservación de los recursos naturales (Geneletti y Van Duren 2008) y diferenciar aquéllas que se enmarcan en un desarrollo productivo vinculados a fines energéticos (García et al. 2006). Ello contribuye a armonizar los mecanismos para la protección de los hábitats, las especies y el uso humano sostenible de áreas de importancia o sensibles como patrimonio natural o cultural. Por esta razón, la zonificación territorial requiere de la evaluación de varios atributos de un área en función de objetivos múltiples (Geneletti y Van Duren 2008) dado que, adicionalmente, ésta es utilizada como un instrumento para abordar las cuestiones de seguridad o equipamiento, minimizando los conflictos en zonas que compiten en una base espacial o temporal (Boyesa et al. 2007).

Bajo las actuales directrices presentes en el país y en el plano internacional, se advierte que los trabajos realizados en esta temática, deben contextualizarse con un enfoque de manejo de cuenca hidrográfica, unidad que presenta una compleja interacción entre componentes naturales, los sistemas económicos y sociales que controlan los recursos naturales, los cuales juegan un papel fundamental en la ordenación del territorio (Mardones y Vargas 2005). Diversos autores indican que la aplicación de criterios de planificación territorial facilitarían la obtención de una zonificación espacial o la identificación de unidades homogéneas sobre las cuales profundizar los análisis al interior de la cuenca (Ontivero et al. 2008). Esta forma de configurar el territorio, se sostiene sobre el interés de conocer la disponibilidad de recursos naturales que aporten en el desarrollo de un área determinada (Gómez-Orea 2007), entendido esto como aquéllos que son necesarios de conservar mediante una adecuada distribución de las actividades en el territorio (Geneletti y Van Duren 2008).

La biomasa forestal como recurso natural ha mostrado mayor atención desde el punto de vista de la planifica- ción para generar desarrollo en materia energética (García et al. 2006, Alaejos y Rosa 2008). Masera et al. (2006), señalan que cuando se utiliza de manera sostenible, este elemento representa una fuente de energía renovable ampliamente disponible. De ahí que generar mapas de biomasa para estos fines es necesario para apoyar la gestión sostenible de los bosques (Leboeuf et al. 2007), y de este modo avanzar en la identificación y zonificación de áreas que produzcan una oferta constante de biomasa residual forestal generada post manejo (corteza, hojas, trozos de madera, etc.). Esto permitiría plantear un escenario de viabilidad económica en un proyecto de suministro energético que considere todos los procesos y acciones en el marco de la gestión territorial. Una aplicación rápida para alcanzar la zonificación de este recurso se puede conseguir integrando los SIG y percepción remota (Beccali et al. 2009). Dan constancia de ello, múltiples trabajos efectuados tanto en Europa, África, Asia y en menor grado en Sudamérica, en donde se han identificado a nivel de prospección zonas de aprovechamiento energético, así como superficies y sitios óptimos para la generación de electricidad a base de biomasa forestal residual (Byrne et al. 2007, MacFarlane 2008).

El requerimiento de información territorial para el sector de la biomasa residual forestal (BRF), toma importancia cuando se consideran las nuevas políticas de fomento energético de fuentes renovables lo que se materializa en la gradual incorporación de las energía renovables no convencionales (ERNC) en el mercado europeo, en donde se espera que su consumo sea cercano al $20 \%$ de la generación eléctrica para el año 2020. De esta forma, también se espera contribuir a la conservación de la energía, a la eficiencia en su uso y el reemplazo de combustibles fósiles para combatir el cambio climático (Lattimore et al. 2009).

Bajo este escenario global, Chile presenta un carácter de importador neto de energía, usando recursos energéticos no renovables, a lo cual se suma una demanda eléctrica que aumenta a una tasa anual del $6,7 \%$ y tan sólo una capacidad instalada de generación eléctrica del 3,1 \% para el año 2007 con energías renovables no convencionales (biomasa, eólica y pequeña hidráulica) (CNE y GTZ 2009). Este escenario genera incertidumbre para las inversiones nacionales o extranjeras que pretenden abastecerse de energías amigables con el medio ambiente, condicionando la materialización de proyectos y nuevos negocios en esta línea. Dicha situación, constituye una oportunidad para zonificar la biomasa residual forestal en el territorio con fines energéticos (Alaejos y Rosa 2008). Más aún, si se considera el potencial de energía renovable de Chile a través de la biomasa con un área aprovechable cercana a los nueve millones de hectáreas de bosque (INFOR 2009). Considerando que la energía es un elemento articulador en los territorios, se plantea como hipótesis que en la cuenca del lago Ranco, existe una extensa superficie de bosque que puede aportar suficiente biomasa residual forestal como alternativa de producción energética en zonas rurales. Por lo cual, el objetivo del presente estudio se centra 
en zonificar áreas potenciales de extracción y manejo de la biomasa residual forestal en la cuenca del lago Ranco mediante la aplicación e integración de herramientas tecnológicas, como los SIG y la percepción remota. Esto permitiría generar una base de información territorial sobre la cual se profundicen trabajos posteriores y se valorice la biomasa residual forestal para fines energéticos.

\section{MÉTODOS}

Área de estudio. El área de estudio corresponde a la cuenca del lago Ranco la cual se ubica entre las coordenadas $72^{\circ}$ $32^{\prime}$ O y $40^{\circ} 40^{\prime} \mathrm{S}$ en el sector suroriental de la provincia del Ranco, en la región de Los Ríos (figura 1). Ésta posee una superficie de 384.349 ha, limita al norte con las comunas de Panguipulli y Los Lagos, al sur con la comuna de Río Bueno, al oeste con las comunas de La Unión y Paillaco, y al este con la Cordillera de Los Andes. Recibe como afluente los ríos Iculpe, Muchi, Caunahue, Calcurrupe, Nilahue, Quimán, Coique y Riñinahue.

En la cuenca se distinguen dos climas, el clima templado lluvioso con influencia mediterránea ( $\mathrm{Cfsb}$, según la clasificación de Koeppen) y el clima de montaña. Morfológicamente, los lagos Maihue, Puyehue y Rupanco, cercanos al lago Ranco, denotan un antecedente glacial que ha

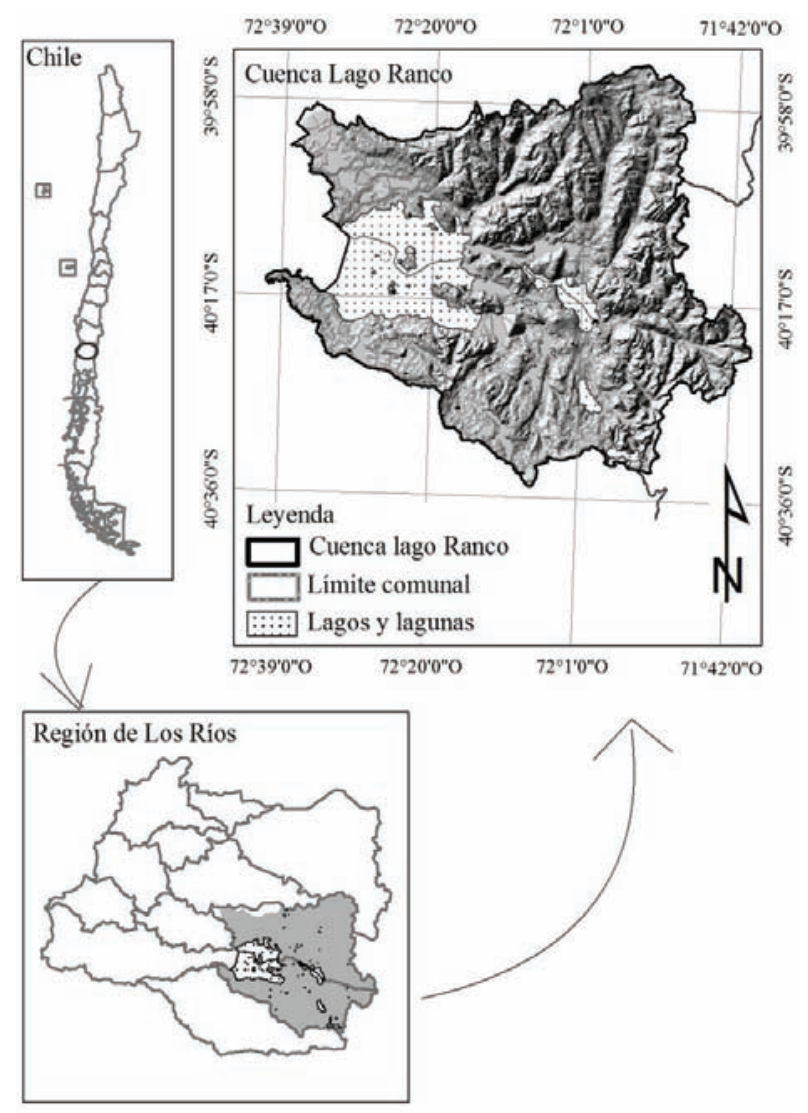

Figura 1. Área de estudio. Study area. sido preservado por morrenas glaciales. Desde la morrena caen hacia la Depresión Intermedia planos inclinados fuertemente sometidos a la acción erosiva de las aguas de esteros y arroyos.

Desde el punto de vista productivo, en la unidad destacan cuatro actividades relevantes en la matriz económica-productiva, los cuales son: el sistema agropecuario, el turismo, el sistema forestal y el sistema hidrobiológico (pesca y acuicultura).

El uso de suelo en la cuenca se caracteriza por una alta proporción de bosque nativo, el cual representa el 51 $\%$ de su superficie. En esta categoría los tipos forestales coigüe-raulí-tepa, lenga, roble-raulí-coigüe y siempreverde corresponden a los sistemas más representativos. Otras categorías de importancia son los cuerpos de agua con $13 \%$ y praderas con $10 \%$ de la superficie. Por su parte, la categoría menos representada son las plantaciones forestales con sólo el 0,6 \% (Peña-Cortés et al. 2009).

Zonificación de áreas con potencial energético con base en la biomasa forestal residual. Se utilizó como información cartográfica de referencia cartas topográficas a escala 1:50.000 del IGM (Instituto Geográfico Militar) con la finalidad de analizar el área geográfica a integrar, además de una imagen de satélite ASTER nivel 1A, de septiembre de 2005, multiespectral de resolución $15 \mathrm{~m}$ para la región del espectro visible e infrarrojo cercano (VNIR). Para actualizar la superficie de bosque se utilizó una metodología estándar para la extracción del NDVI con las bandas contenidas en el espectro visible e infrarrojo cercano (bandas 2 y 3). Posteriormente, esta imagen fue georreferenciada con el módulo Ortho Engine del software PCI Geomatics y proyectada en el sistema Universal Transversal de Mercator (UTM). Para la modelación espacial y edición cartográfica se utilizaron los softwares ArcView ${ }^{\mathrm{TM}}$ V3.2 y ArcGis $^{\mathrm{TM}}$ V8.2. La zonificación del potencial energético se realizó atendiendo a cinco criterios de restricción de manejo de biomasa forestal residual: (1) superficies con pendientes mayores a $60 \%$ (MINAGRI 2008), (2) barrera de 30 metros a cursos de agua, (3) presencia de áreas silvestres protegidas por el Estado, (4) barrera de 100 metros en áreas de riesgo natural (derrumbes y deslizamientos) definidos en el plan de ordenamiento territorial de la cuenca del lago Ranco (Peña-Cortés et al. 2009) y (5) el estado de desarrollo del bosque en el cual se incluyó sólo bosque adulto y mixto, por cuanto sólo en ellos se presentan registros de manejo en la cuenca.

Estimación de la energía disponible en la biomasa forestal residual con base en su poder calorifico. El potencial energético de la biomasa forestal residual (restos de hojas, ramas, trozos de madera, lampazos) se estimó en base a un muestreo efectuado en cuatro unidades de vegetación: tipo forestal coigüe-raulí-tepa, roble-raulí-coigüe, siempreverde y plantaciones forestales (mezcla de Pinus radiata D. Don con Eucalyptus spp.). Cabe mencionar que en el 
marco de este análisis el tipo forestal lenga quedó excluido, ya que no existieron registros de manejo en la cuenca ${ }^{1}$. En estas unidades vegetacionales se identificaron áreas cosechadas, de acuerdo a la información contenida en los planes de manejo facilitados por la Corporación Nacional Forestal (CONAF). En cada unidad de vegetación se consideró un sitio de muestreo, el cual se seleccionó a base de dos criterios: (1) áreas de cosecha reciente identificadas por CONAF y (2) accesibilidad vial con autorización de ingreso al predio. En cada sitio se efectuó un transecto de $10 \mathrm{~m}$ ubicado en dirección norte-sur, sobre el cual se distribuyeron cinco cuadrantes de $1 \mathrm{~m}^{2}$ donde se obtuvieron muestras de biomasa residual de la cosecha. Se empleó una motosierra para trozar aquellos residuos forestales de amplio volumen y un rastrillo para recoger aquéllos de menor dimensión, limpiando completamente el cuadrante. En cada cuadrante se pesó la totalidad de la biomasa. Luego se obtuvieron muestras de $500 \mathrm{~g}$ por sitio, las cuales se rotularon y se enviaron al laboratorio de la Unidad de Desarrollo Tecnológico de la Universidad de Concepción, para obtener el poder calorífico de los residuos mediante el método descrito en el manual del equipo "calorímetro con bomba de oxígeno modelo 1341"; además, se obtuvo el porcentaje de humedad a través del ensayo adaptado de la norma chilena NCh176/1 y la determinación del porcentaje de cenizas según método ASTM D1102-56 Ash in wood. El valor potencial real de energía se obtuvo al aplicar la corrección porcentual considerando la humedad de la muestra, la cual expresa la eficiencia térmica de la biomasa (Gallegos 2009).

Con los antecedentes levantados en terreno y los resultados de laboratorio se extrapolaron los valores de energía a una unidad espacial mínima cartografiable (una hectárea). Posteriormente, se realizó una operación aritmética asistido por el programa ArcView GIS ${ }^{\mathrm{TM}}$ V3.2 en donde se multiplicó el coeficiente de energía por la superficie óptima definida en cada unidad vegetacional consiguiendo asociar a cada una de éstas el potencial energético de la biomasa residual forestal, expresada en kilowatt-hora por hectárea $(\mathrm{kWh} / \mathrm{ha})$.

\section{RESULTADOS}

Zonificación del potencial energético con base en la biomasa forestal residual en la cuenca del lago Ranco. Las cuatro unidades vegetacionales alcanzaron una superficie de 139.023 ha de bosque (tipos forestales coigüe-raulí-tepa, roble-raulí-coigüe, siempreverde y plantaciones forestales). Posteriormente, luego de aplicar los cinco criterios de restricción previamente definidos se obtuvo una superficie potencial de manejo de la biomasa forestal residual de 45.555 ha para uso energético, lo que equivale al 32,8 \% del total de la superficie de bosque en las unidades vegetacionales (figura 2).

\footnotetext{
${ }^{1}$ M Lobos. Extensionista forestal, Corporación Nacional Forestal, comuna de Futrono. Comunicación personal, 2008.
}

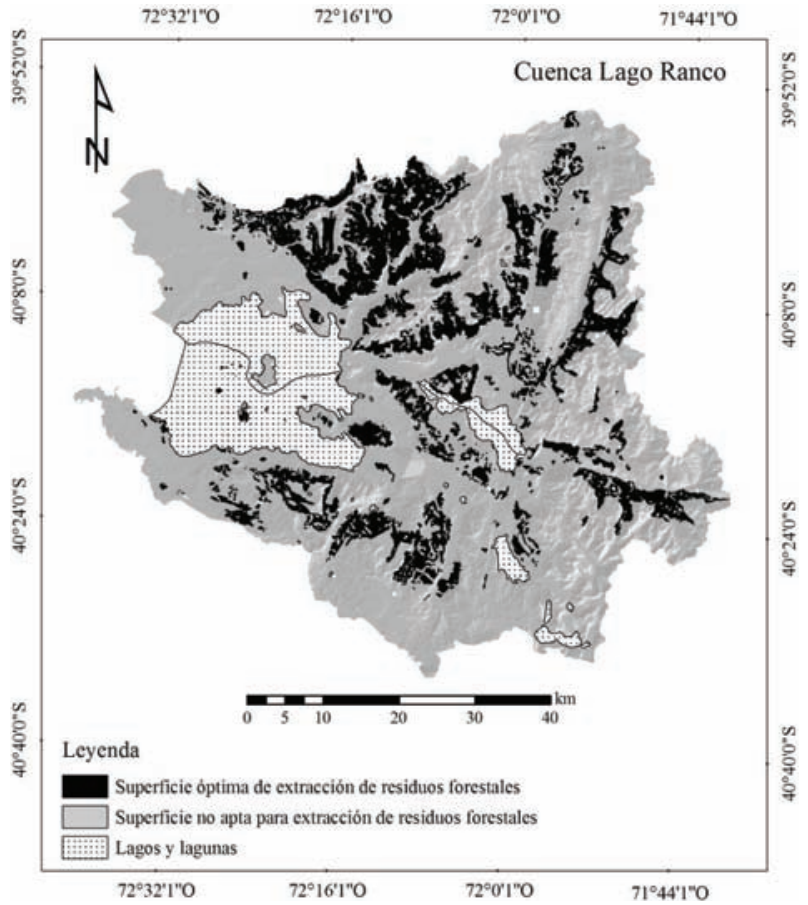

Figura 2. Superficie potencial de manejo (área obscura) de la biomasa forestal residual para uso energético en la cuenca del lago Ranco.

Potential surface of handling residual forest biomass in the watershed of Ranco Lake.

Considerando las unidades de vegetación, el tipo forestal coigüe-raulí-tepa con 28.459 ha representó el 63 $\%$ de la superficie disponible para manejo energético, mientras que roble-raulí-coigüe y el tipo forestal siempreverde con 7.516 ha y 9.073 ha representaron el 17 $\%$ y $19 \%$, respectivamente. Por su parte, las plantaciones forestales destacan por su baja participación con 507 ha equivalente al $1 \%$ de área afecta a manejo (cuadro 1).

La medición de la biomasa forestal residual en terreno mostró que la mayor disponibilidad se presentó en el tipo forestal siempreverde con $484 \mathrm{t} / \mathrm{ha}$, seguido en orden decreciente de magnitud por el tipo forestal robleraulí-coigüe con $190 \mathrm{t} / \mathrm{ha}$. Las plantaciones forestales representaron la tercera magnitud con una acumulación de biomasa de $186 \mathrm{t} / \mathrm{ha}$. Por su parte, el tipo forestal coigüeraulí-tepa representó la disponibilidad de residuos más baja con $170 \mathrm{t} / \mathrm{ha}$.

Energía disponible en la biomasa forestal residual según unidad de vegetación evaluada. El análisis de laboratorio de la muestra de biomasa forestal residual del tipo forestal coigüe-raulí-tepa, a pesar de contener el mayor porcentaje de humedad (44\%), fue la de mayor poder calorífico alcanzando $4.886 \mathrm{kcal} / \mathrm{kg}$. Por su parte, la muestra de biomasa con menor poder calorífico fue la obtenida en plantaciones forestales con $4.489 \mathrm{kcal} / \mathrm{kg}$. A su vez, esta muestra generó aproximadamente un $8 \%$ de cenizas, cifra que es una de las más altas dentro de los resultados. 
El poder calorífico obtenido, expresado en kilocalorías por kilogramo de biomasa forestal residual, se transformó a kilowatt-hora $(\mathrm{kWh})$ por hectárea, de este modo la energía disponible puede llegar a valores muy altos como ocurre para el tipo forestal siempreverde con $2.612 \mathrm{kWh} / \mathrm{ha}$. Sin embargo, tomando como antecedente base la relación entre eficiencia térmica respecto del contenido de humedad de la biomasa, el potencial calorífico se reduciría a un $68 \%$ (Gallegos 2009), esto considerando el valor promedio de la humedad encontrada en las muestras obtenidas en campo (32\%), por lo cual el valor real del poder calorífico en el caso de esta unidad alcanzaría $1.776 \mathrm{kWh} / \mathrm{ha}$, mientras que el tipo forestal roble-raulí-coigüe llegaría a $685 \mathrm{kWh} / \mathrm{ha}$. Por su parte, el tipo forestal coigüe-raulí-tepa y las plantaciones forestales presentarían $659 \mathrm{kWh} / \mathrm{ha}$ y $658 \mathrm{kWh} / \mathrm{ha}$, respectivamente, mostrando el menor potencial energético (cuadro 2).

La dinámica actual de cosecha en la cuenca, principalmente de bosque nativo, es cercana a las $1.200 \mathrm{ha} / \mathrm{año}$, lo cual se traduce en una generación anual de $1.133 .400 \mathrm{kWh}$ tomando como promedio un potencial energético de 944,5 $\mathrm{kWh} / \mathrm{ha}$. Tal cantidad de energía cubriría la demanda de 472 hogares por año tomando como base un consumo de $2.400 \mathrm{kWh} /$ año en una comuna de la provincia de Valdivia (Rocha 2009).

\section{DISCUSIÓN}

Este trabajo da cuenta de que la zonificación se facilita cuando se considera una base actualizada de información territorial que exprese un adecuado conocimiento del espacio geográfico. Destacan en este proceso la integración de herramientas que permiten realizar una adecuada organización de las políticas y la proyección espacial del desarrollo considerando aspectos sociales, económicos, ambientales y culturales que garanticen una adecuada calidad de vida de la población (Asher y Ojeda 2009).

La comprensión de la dinámica territorial permite contar con una base confiable para concretar inversión tanto pública como privada, de tal modo que facilite aprovechar las ventajas de los incipientes clusters (basados principalmente en recursos naturales), para generar entornos que incentiven la innovación y, que por medio del aumento de la productividad primaria, permitan transformar las ventajas estáticas en dinámicas.

Bajo este escenario el presente trabajo se alinea en un contexto desarrollista de carácter prospectivo, considerando como una oportunidad la utilización de biomasa forestal residual de modo sustentable (considerando restricciones como la pendiente del terreno, protección de cursos de agua, exclusión del sistema nacional de áreas silvestres

Cuadro 1. Superficie potencial de manejo de biomasa forestal residual por unidad de vegetación en la cuenca del lago Ranco. Potential surface of handling residual forest biomass for vegetation units assessed in the watershed of Ranco Lake.

\begin{tabular}{lccc}
\hline \multicolumn{1}{c}{$\begin{array}{c}\text { Unidad de vegetación } \\
\text { evaluada }\end{array}$} & $\begin{array}{c}\text { Superficie total } \\
\text { (ha) }\end{array}$ & $\begin{array}{c}\text { Superficie con restricción } \\
\text { de manejo (ha) }\end{array}$ & $\begin{array}{c}\text { Superficie potencial a } \\
\text { manejar (ha) }\end{array}$ \\
\hline Coigüe-raulí-tepa & 88.122 & 59.663 & 28.459 \\
Siempreverde & 18.654 & 9.581 & 9.073 \\
Roble-raulí-coigüe & 31.202 & 23.687 & 7.516 \\
Plantaciones forestales & 1.044 & 537 & 507 \\
\hline Total & $139.023^{*}$ & 93.468 & 45.555 \\
\hline
\end{tabular}

* Esta superficie no considera el tipo forestal lenga.

Cuadro 2. Potencial energético de la biomasa forestal residual por unidad de vegetación en la cuenca del lago Ranco. Energy potential of residual forest biomass by vegetation units assessed in the watershed of Ranco Lake.

\begin{tabular}{lcccrrrr}
\hline $\begin{array}{c}\text { Unidad de vegeta- } \\
\text { ción evaluada }\end{array}$ & $\begin{array}{c}\text { Poder } \\
\text { calorífico } \\
(\mathrm{kcal} / \mathrm{kg})\end{array}$ & $\begin{array}{c}\text { Con- } \\
\text { versión } \\
(\mathrm{kWh})^{*}\end{array}$ & $\begin{array}{c}\text { Biomasa } \\
\text { forestal } \\
\text { residual } \\
(\mathrm{t} / \mathrm{ha})^{* *}\end{array}$ & $\begin{array}{c}\text { Energía } \\
\text { disponible } \\
(\mathrm{MWh} / \mathrm{ha})\end{array}$ & $\begin{array}{c}\text { Potencial } \\
\text { energético real } \\
(\mathrm{MWh} / \mathrm{ha}) * * *\end{array}$ & $\begin{array}{c}\text { Superficie } \\
\text { potencial a } \\
\text { manejar (ha) }\end{array}$ & $\begin{array}{c}\text { Potencial } \\
\text { energético } \\
\text { total }(\mathrm{MWh} / \\
\mathrm{UVE})^{\#}\end{array}$ \\
\hline Coigüe-raulí-tepa & 4.886 & 5,7 & 170 & 969 & 659 & 28.459 & 18.754 .481 \\
Siempreverde & 4.631 & 5,4 & 484 & 2.612 & 1.776 & 9.073 & 16.113 .648 \\
Roble-raulí-coigüe & 4.577 & 5,3 & 190 & 1.007 & 685 & 7.516 & 5.148 .460 \\
Plantación forestales & 4.489 & 5,2 & 186 & 967 & 658 & 507 & 333.606 \\
\hline
\end{tabular}

$* 1 \mathrm{kcal}=0,001163 \mathrm{kWh}$ (Rocha 2009). **Incluye restos de hojas, ramas, trozos de madera y lampazos. ${ }^{* * *}$ Considera una eficiencia térmica de 68

$\%$ (Gallegos 2009). "UVE: unidad vegetacional evaluada. 
protegidas del Estado, áreas de riesgos naturales y estado de desarrollo del bosque), proveniente en gran parte del manejo del bosque nativo. En este contexto, cabe mencionar que esta zonificación territorial se ha abordado de forma preliminar, aunque la información estratégica acerca de la disponibilidad de biomasa en Chile y en sus regiones (INFOR 2009), bajo un supuesto de manejo forestal, podrían generar una oferta sustentable de biomasa forestal residual.

Desde el punto de vista metodológico este estudio contiene elementos de carácter estándar en la operación de los sistemas de información geográfica y percepción remota, y el procesamiento de información territorial, lo cual permite un proceso de evaluación rápida, de baja complejidad y de bajo costo para identificar elementos de interés que puedan generar un desarrollo económico en la cuenca. Cabe destacar que, por su condición de aplicación simplificada, esta metodología entrega una base de decisión sólo de carácter orientador en la temática energética de la cuenca del lago Ranco.

Bajo un esquema de aprovechamiento de la biomasa forestal residual, la aplicación aquí planteada se justifica bajo la normativa señalada por la ley de bosque nativo $\mathrm{N}^{\circ}$ 20.283 (MINAGRI 2008) y la actual condición del bosque que, por sus características, se calificaría como bosque productivo comercial, lo cual permite el manejo integrado de éste con base en elementos de ordenamiento forestal y planes de manejo. Además, se favorecen iniciativas vinculadas al decreto ley $\mathrm{N}^{\circ} 701$ (MINAGRI 1974), como el repoblamiento de especies cosechadas y permite abordar acciones de gestión territorial con pequeños y medianos propietarios.

En cuanto a las cantidades de biomasa forestal residual, si sólo se considera la superficie de los tres tipos forestales, se observó que el promedio obtenido en el marco de este trabajo fue de $258 \mathrm{t} / \mathrm{ha}$, cantidad superior a los registros obtenidos por el INFOR (2009) en mediciones realizadas entre los años 2000-2008 en distintas parcelas de muestreos las que en promedio entregaron $115 \mathrm{t} / \mathrm{ha}$. La marcada diferencia en masa de residuos puede ser atribuida entre otros a las condiciones de sitio, estado de desarrollo del bosque y mayor número de muestreos realizados por la entidad estatal.

Respecto al valor energético en las muestras de biomasa residual forestal, éste se aproxima al promedio de 4.525 $\mathrm{kcal} / \mathrm{kg}$ obtenidos por Nuñez-Regueira et al. (1996), en un estudio que incluyó igualmente corteza, frutos, ramas y hojas de coníferas y latifoliadas. Bhatt y Tomar (2002) obtuvieron resultados en promedio mayor, alcanzando máximos que superaron los $5.000 \mathrm{kcal} / \mathrm{kg}$, situación que los autores atribuyen a la alta densidad (promedio $0,91 \mathrm{~g} / \mathrm{cm}^{3}$ ) y bajo contenido de cenizas (promedio 2,7\%).

Considerando la superficie potencial de la cuenca del lago Ranco que cumple con los requerimientos propuestos en el marco del estudio (restricciones territoriales) y el supuesto de que la superficie en promedio cosechada (1.200 ha/año) fuese constante en el tiempo, el primer ciclo completo de utilización de la biomasa residual forestal podría alcanzar teóricamente 37,9 años.

Luego del primer ciclo podrían encontrarse bosques de 38 años compuestos de roble (Nothofagus obliqua (Mirb.) Oerst.) con un volumen de $314,1 \mathrm{~m}^{3} /$ ha considerando un crecimiento de $8,2 \mathrm{~m}^{3} /$ ha y por año tal como se constata en Nilahue (localidad inserta en el área de estudio) (Donoso 1993). Esta proyección podría cubrir el desarrollo de una central termoeléctrica a base de una potencia de 1,1 MW/ año por un periodo cercano a los 38 años, abastecida con los residuos forestales provenientes de la superficie anual cosechada en la cuenca del lago Ranco. Esta generación eléctrica tendría un costo aproximado de US\$ 3.600 .000 por MW/año según análisis realizado por Soto y Werner (2009) para implementar una alternativa de generación eléctrica a partir de residuos de biomasa forestal.

En la actualidad existen iniciativas asociadas a la biomasa en múltiples ámbitos de estudio, como por ejemplo para estimar cantidad de carbono, la producción energética con base en la biomasa fustal (Rocha 2009) y otras iniciativas desarrolladas por entidades estatales chilenas con apoyo extranjero (CNE et al. 2007), en donde se analiza la producción de electricidad desde residuos forestales provenientes de aserraderos a nivel regional y nacional. Según lo anterior, estudios específicos a nivel de biomasa residual desde bosques no han sido abordados en la actualidad, lo cual abre una oportunidad para desarrollar trabajos en esta línea que busquen utilizar los pasivos ambientales de los bosques con el fin de fortalecer el potencial energético.

Finalmente, este trabajo muestra datos referenciales útiles para estimar la energía aprovechable desde la biomasa forestal residual en la cuenca de lago Ranco, temática abordada bajo un enfoque desarrollista considerando un manejo sustentable del bosque nativo y, a su vez, teniendo presente la visión de la OECD (2009) que señala que al aumentar el crecimiento regional a través de políticas territoriales específicas para cada contexto, no sólo se fortalecerá el crecimiento nacional, sino que también la cohesión territorial. Esta última constituye un elemento importante en una economía abierta y sustentable, lo cual es totalmente complementario con el enfoque de integración gradual de nuevas políticas que incentiven el uso de fuentes de energías renovables no convencionales.

\section{CONCLUSIONES}

La información territorial es fundamental para llevar a cabo un correcto proceso de toma de decisiones. En este sentido, el procesamiento de datos asistido por sistemas de información geográfica y percepción remota utilizado en este estudio, entrega aproximaciones útiles para la toma de decisiones en el sector energético, las cuales son replicables y adaptables a escala local, regional y nacional, pues se rigen bajo metodologías estándar de análisis espacial y, a su vez, presentan un marco referencial a base 
de otros estudios existentes para el sector energético.

En un contexto exploratorio, la unidad de vegetación coigüe-raulí-tepa presenta ventajas comparativas en la cuenca desde el punto de vista de la superficie potencial de manejo y el poder calorífico contenido en la biomasa forestal residual. Por lo que esta unidad se presenta como la mejor alternativa al momento de evaluar opciones de aprovechamiento de la biomasa forestal residual con fines de generación energética.

Con la actual disponibilidad de biomasa residual forestal en la cuenca y bajo el escenario de crisis energética, es necesario ampliar este tipo de investigaciones para evaluar la factibilidad técnico-económica de implementar un mecanismo que genere energías renovables no convencionales, valorando este recurso bajo un esquema de manejo sustentable del bosque nativo.

\section{AGRADECIMIENTOS}

A Jimmy Pincheira, por el apoyo en la edición y revisión crítica del manuscrito. La presente investigación contó con el financiamiento de la Dirección General de Investigación y Postgrado de la Universidad Católica de Temuco, proyecto DGIUCT N² 2007-DGI-TP-01.

\section{REFERENCIAS}

Alaejos J, Rosa M. 2008. Estudio del potencial de biomasa en el término municipal de Cartaya (Huelva). Boletín del CIDEU 5: $67-83$

Asher K, D Ojeda. 2009. Producing nature and making the state: Ordenamiento territorial in the Pacific lowlands of Colombia. Geoforum 40: 292-302.

Beccali M, P Columbab, V D'Albertia, V Franzitta. 2009. Assessment of bioenergy potential in Sicily: A GIS-based support methodology. Biomass and Bioenergy 33:79-87.

Belmonte S, V Nuñez, J Viramonte, J Franco. 2008. Potential renewable energy resources of the Lerma Valley, Salta, Argentina for its strategic territorial planning. Renewable and Sustainable Energy Reviews 13: 1475-1484.

Bhatt B, J Tomar. 2002. Firewood properties of some Indian mountain tree and shrub species. Biomass and Bioenergy 23: $257-260$

Boyesa S, M Elliotta, S Thomsona, S Atkinsb, P Gilliland. 2007. A proposed multiple-use zoning scheme for the Irish Sea. An interpretation of current legislation through the use of GIS-based zoning approaches and effectiveness for the protection of nature conservation interests. Marine Policy 31: $287-298$

Byrne J, A Zhou, B Shen, K Hughes. 2007. Evaluating the potential of small-scale renewable energy options to meet rural livelihoods needs: A GIS- and lifecycle cost-based assessment of Western China's options. Energy Policy 35: 4391-4401.

Chen N, H Li, L Wang. 2008. A GIS-based approach for mapping direct use value of ecosystem services at a county scale: Management implications. Ecological and Economics 68: 2768-2776.
CNE (Comisión Nacional de Energía, CL), INFOR (Instituto Forestal, CL), GTZ (Deutsche Gesellschaft für Technische Zusammenarbeit, DE). 2007. Disponibilidad de residuos madereros. Residuos de la industria primaria de la madera - Disponibilidad para uso energético. Consultado 07 jun. 2010. Disponible en http://www.cer.gov.cl/documentos/

CNE (Comisión Nacional de Energía, CL) y GTZ (Deutsche Gesellschaft für Technische Zusammenarbeit, DE). 2009. Las energías renovables no convencionales en el mercado eléctrico chileno. Consultado 09 jul. 2010. Disponible en http://www.gtz.de/de/dokumente/gtz2009-sp-erncmercado-electrico-chileno.pdf

Donoso C. 1993. Bosques templados de Chile y Argentina. Variación, estructura y dinámica. Ecología forestal. Santiago, Chile. Universitaria. 484 p.

Gallegos R. 2009. Cuantificación y utilización de los residuos de cosecha forestal, específicamente de tocones de una plantación de Eucalytus nitens (Dean et Maiden), para la producción de energía renovable, en la Región de Los Ríos. Tesis Ingeniero Forestal. Valdivia, Chile. Facultad de Ciencias Forestales, Universidad Austral de Chile. 60 p.

García A, F Pérez-Cabello, J De la Riva. 2006. Evaluación de los recursos de biomasa residual forestal mediante imágenes del satélite Landsat y SIG. GeoFocus 6: 205-230.

Geneletti D, I Van Duren. 2008. Protected area zoning for conservation and use: A combination of spatial multicriteria and multiobjective evaluation. Landscape and Urban Planning 85: 97-110.

Gómez-Orea D. 2007. Ordenación del territorio. Una aproximación desde el medio físico. Madrid, España. Editorial Instituto Tecnológico Geominero de España y Ed. Agrícola Española. 704 p.

INFOR (Instituto Forestal, CL). 2009. Los recursos forestales en Chile. Informe final: Inventario continuo de bosques nativos y actualización de plantaciones forestales. Ministerio de Agricultura, Gobierno de Chile. Consultado 13 may. 2010. Disponible en http://www.infor.cl/en/publicacionesinfor/109-informes-contrato-de-deempeno/327-inventariocontinuo-de-bosques-nativos-y-actualizaciones-deplantaciones-forestales.html

Lattimore B, C.T Smith, B Titus, I Stupak, G Egnell. 2009. Environmental factors in woodfuel production: Opportunities, risks, and criteria and indicators for sustainable practices. Biomass and bioenergy 33: 13211342.

Leboeuf A, A Beaudoin, R Fournier, L Guindon, J Luther, M Lambert. 2007. A shadow fraction method for mapping biomass of northern boreal black spruce forests using QuickBird imagery. Remote Sensing of Environment 110: 488-500.

MacFarlane D. 2008. Potential availability of urban wood biomass in Michigan: Implications for energy production, carbon sequestration and sustainable forest management in the U.S.A. Biomass and Bioenergy 33: 628-634.

Mardones M, J Vargas. 2005. Efectos hidrológicos de los usos eléctrico y agrícola en la cuenca del río Laja (Chile centrosur). Revista de geografia Norte Grande 33: 89-102.

Masera O, A Ghilardi, R Drigo, M Trossero. 2006. WISDOM: A GIS-based supply demand mapping tool for woodfuel management. Biomass and Bioenergy 30: 618-637.

MINAGRI (Ministerio de Agricultura, CL). 1974. Decreto 
ley $\mathrm{n}^{\mathrm{o}}$ 701. Gobierno de Chile. Consultado 11 de ene. 2010. Disponible en http://www.leychile.cl/ Navegar?idNorma $=148500$

MINAGRI (Ministerio de Agricultura, CL). 2008. Ley nº 20.283 sobre recuperación del bosque nativo y fomento forestal. Gobierno de Chile. Consultado 11 ene. 2010. Disponible en http://www.leychile.cl/Navegar?idNorma=274894\&buscar $=$ ley + bosque + nativo

Nuñez-Requeira L, J Rodríguez, P Castiñeiras. 1996. Calorific values and flammability of forest species in Galicia. Coastal and hillside zones. Bioresource Technology 57: 283-289.

OECD (Organización para la Cooperación Económica y el Desarrollo, CL). 2009. Estudio Territorial OECD-Chile. Consultado 29 dic. 2009. Disponible en http://www.oecd.org/document/25/0,3343, fr_33873108_39418658_43391385_1_1_1_1,00.html

Ontivero M, J Martínez-Vega, V González, P Echavarría. 2008. Propuesta metodológica de Zonificación Ambiental en la sierra de altomira mediante sistemas de información geográfica. GeoFocus 8: 251-280.

Peña-Cortés F, M Escalona-Ulloa, G Rebolledo, P Gutiérrez, O Almendra, J Pincheira-Ulbrich, O Torres-Álvarez, E Fernández. 2009. Contribución de las tecnologías geoespaciales en el ámbito nacional. In Mena-Frau $\mathrm{C}$ ed. Tecnologías geoespaciales: Experiencias aplicadas al estudio y gestión del territorio. Santiago, Chile. Editorial Servicio Aerofotogramétrico de la Fuerza Aérea de Chile (SAF-FACH). p. 25-36.

Rocha P. 2009. Oferta disponible de biomasa forestal bajo restricciones de protección y propiedad para la producción de energía eléctrica en la comuna de Corral. Tesis Ingeniero Forestal. Valdivia, Chile. Facultad de Ciencias Forestales, Universidad Austral de Chile. 51 p.

Soto I, L Werner. 2009. Evaluación técnica y económica de una central termoeléctrica en la Región de Los Ríos. Tesis Ingeniero Mecánico. Valdivia, Chile. Facultad de Ciencias de la Ingeniería, Universidad Austral de Chile. 106 p.

Recibido: 11.08 .10

Aceptado: 07.01.11 